\title{
Illuminating Nucleon Structure Through Polarized Proton-Proton Collisions at STAR
}

\author{
J. L. Drachenberg* \\ Lamar University \\ E-mail: James.Drachenberg@lamar.edu
}

\begin{abstract}
During the past decade, the experiments of the RHIC spin program have provided critical insight into the spin structure of the nucleon, in particular shedding light on the roles played by gluon and sea-quark helicity. In the forthcoming RHIC run, attention will be devoted to transverse-spin phenomena. Over the last decade, theoretical and experimental engagement of this oft-challenging subject has unlocked tantalizing opportunities for new insight into nucleon structure, e.g. with higher dimensions in partonic momentum space. The STAR experiment continues this exploration through an array of measurements from high-energy polarized-proton collisions. Among these studies are the production of weak bosons, azimuthal distributions of hadrons within jets, dihadron correlations, and particle production at large pseudorapidity. Recent breakthroughs may illuminate further longstanding questions: Do factorization and universality extend to the transverse-momentum-dependent (TMD) picture in proton-proton collisions? How do TMD functions evolve with changing kinematics? Beyond existing probes, future measurements will enable even wider frontiers in understanding QCD and nucleon structure.
\end{abstract}

XXV International Workshop on Deep-Inelastic Scattering and Related Subjects

3-7 April 2017

University of Birmingham, $U K$

\footnotetext{
* Speaker.
} 


\section{Introduction}

Spin in particle physics proves to be an incisive tool in opening windows into a deeper understanding of nucleon structure. Global analyses combining results from deep inelastic scattering (DIS), semi-inclusive DIS (SIDIS), and polarized proton-proton $(p+p)$ collisions have recently brought enhanced clarity to the roles of gluon and sea-quark helicity (e.g. Ref. [1]). Global analyses (e.g. Ref. [2,3]) of transverse single-spin asymmetries in charged pions produced in SIDIS and $e^{+} e^{-}$collisions have also enabled the first extractions of transversity through the Collins mechanism [4]. Additional global analyses (e.g. Ref. [5]) have extracted transversity through dihadron "interference fragmentation functions" (IFF) [6]. Polarized $p+p$ collisions provide a novel way to expand the frontier. Such measurements extend the kinematic reach in both $x$ and $Q^{2}$ and also eliminate the $u$-quark dominance that limits constraints that SIDIS data apply to $d$-quark transversity. Furthermore, polarized $p+p$ collisions provide a unique way to probe open questions about TMD factorization and universality. TMD factorization has been proven for SIDIS as well as for Drell-Yan and weak-boson production in polarized $p+p$ collisions (e.g. Ref. [7]). Measurements such as transverse single-spin asymmetries in weak-boson production from polarized $p+p$ probe the well-known "sign-change" expectation [7] between SIDIS and $p+p$ through the Sivers mechanism [8]. The large mass of the boson provides a high $Q^{2}$ scale that should make the asymmetries sensitive to the evolution of the TMDs. On the other hand, TMD factorization is generally expected to be broken for hadronic interactions (e.g. Ref. [9]), for instance in single high- $p_{T}$ meson production. The size of any such factorization breaking is not known. It has recently been argued that the cross section for hadrons within jets produced in $p+p$ does factorize and only depends upon universal TMD fragmentation functions, decoupled from TMD PDFs [10]. In contrast to TMDs, IFF asymmetries persist in the collinear framework of $\mathrm{pQCD}$, where factorization and universality are expected to hold [11]. The combination of data from hadrons within jets and dihadrons from $p+p$ with existing SIDIS and $e^{+} e^{-}$data provide the opportunity for a comprehensive global analyses to address these long-standing theoretical questions.

Over the last decade, the STAR experiment [12] at RHIC has conducted an aggressive program to study transverse spin effects in polarized $p+p$ collisions at both $\sqrt{s}=200$ and $500 \mathrm{GeV}$. The first signatures of transversity in $p+p$ have been seen in charged-pion Collins [13] and IFF [14] asymmetries at $|\eta|<1$ from $2.4 \mathrm{pb}^{-1}$ at $\sqrt{s}=200 \mathrm{GeV}$ collected in 2006. To improve the precision of these measurements, STAR collected $22 \mathrm{pb}^{-1}$ of luminosity from $p+p$ at $\sqrt{s}=200 \mathrm{GeV}$ with $63 \%$ polarization during the 2012 RHIC run. In 2011, STAR integrated an additional $25 \mathrm{pb}^{-1}$ of luminosity from $p+p$ at $\sqrt{s}=500 \mathrm{GeV}$ with $53 \%$ polarization. This dataset not only enables the first measurements of transversity effects at $\sqrt{s}=500 \mathrm{GeV}$, but also the first measurement of transverse single-spin asymmetries in weak boson production.

\section{Analysis}

Detailed descriptions of the analysis techniques and simulation studies for the jet measurements are given in Refs. [15, 16], while those for the IFF measurements are given in Refs. [14, 17, 18]. Jets are reconstructed using the "anti- $k_{T}$ " algorithm [19] with a radius of 0.6 for $\sqrt{s}=200$ 
$\mathrm{GeV}$ or 0.5 for $\sqrt{s}=500 \mathrm{GeV}$ and utilize energy deposition in the BEMC and EEMC as well as charged-particle momenta from STAR's time projection chamber (TPC) [12].

A description of the weak boson analysis is given in Ref. [20]. Events are selected by requiring an isolated high- $p_{T}$ electron or positron determined by energy from the BEMC towers and momenta from the TPC. The full kinematics of the boson are reconstructed by summing the hadronic recoil momenta and employing an event-by-event Monte Carlo correction to compensate for recoil momenta that fall outside the STAR acceptance.

\section{Results}
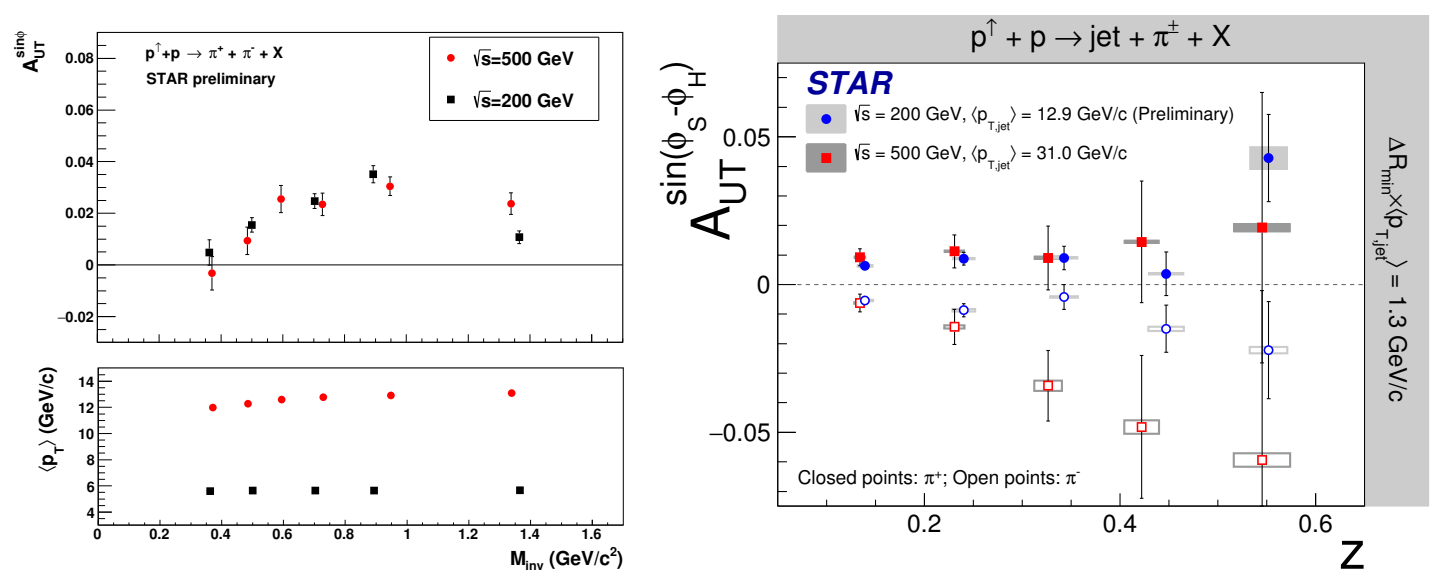

Figure 1: STAR charged-pion IFF [17, 18] (left) and Collins $[15,16]$ (right) asymmetries at $\sqrt{s}=200$ and $500 \mathrm{GeV}$. In both cases, asymmetries are consistent when scaling for $x_{T}$.

Charged-pion IFF [17, 18] and Collins [15, 16] asymmetries from STAR's 2011 and 2012 datasets are presented in Fig. 1. IFF asymmetries are shown as functions of dihadron invariant mass, while the Collins asymmetries are shown versus pion $z$, the fraction of jet momentum carried by the pion. The kinematics are selected such that the $200 \mathrm{GeV}$ and $500 \mathrm{GeV}$ data sample the same range of $x_{T}$. In the case of the Collins asymmetries, the data are also selected to sample the same range of pion momentum transverse to the jet axis, $j_{T}=z \times \Delta R \times p_{T}$, jet, where $\Delta R$ is the angular distance of the pion from the jet axis. For both the IFF and Collins mechanisms, the $200 \mathrm{GeV}$ and $500 \mathrm{GeV}$ asymmetries are consistent for common ranges of $x_{T}$. It is noteworthy that this scaling is seen in an observable surviving in the collinear framework (IFF) and in an observable requiring TMD factorization (Collins) that is generally expected to be broken in hadroproduction.

The IFF asymmetries from STAR's 2006 dataset [14] have been compared with model calculations based upon SIDIS and $e^{+} e^{-}$data [21]. The model calculations show agreement with the STAR data in terms of the dihadron mass dependence. Efforts to include these data in global analyses aimed at extracting transversity have already begun [22].

The STAR Collins asymmetries [15, 16] have been compared with model calculations [24, 25] based upon SIDIS and $e^{+} e^{-}$data. The comparisons for the $\sqrt{s}=500 \mathrm{GeV}$ data [15] are shown in the right-hand panel of Fig. 2. The models assume robust TMD factorization and universality. 

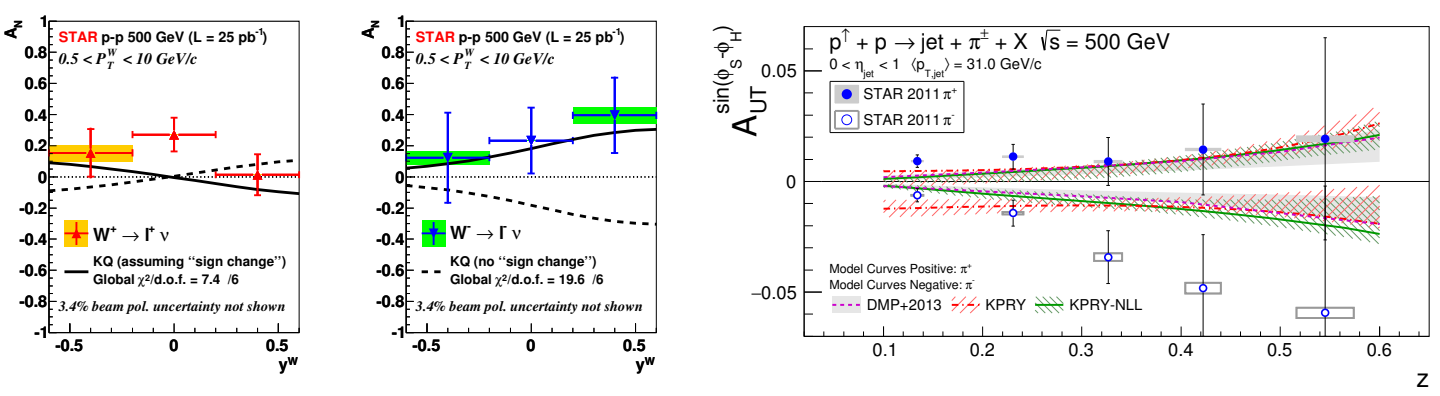

Figure 2: STAR weak boson [20] (left and center) and charged-pion Collins [15] (right) asymmetries at $\sqrt{s}=500 \mathrm{GeV}$ in comparison to model calculations based on SIDIS [23, 24, 25]. Weak boson asymmetries show a strong preference for models that assume the sign-change [7], provided suppression due to TMD evolution is small. Collins asymmetries are consistent with models at the $95 \%$ confidence level showing a slight preference for models assuming no TMD evolution.

Furthermore, the models of Ref. [25] are based on two separate calculations: one assuming no TMD evolution (KPRY) and one assuming TMD evolution up to next-to-leading log (KPRY-NLL). The STAR data are in relatively good agreement with all of the model calculations. As demonstrated by Fig. 2, the $500 \mathrm{GeV}$ data are consistent with all predictions at the $95 \%$ confidence level. This is consonant with the model assumption of universality of the Collins fragmentation function and the assumption of robust TMD factorization for these observables in $p+p$. Between the two KPRY calculations, the $500 \mathrm{GeV}$ data do exhibit a slight preference for the model without TMD evolution. Using the data statistical and systematic uncertainties, a $\chi^{2}$ test yields $\chi^{2}=14.0$ for $v=10$ degrees of freedom without evolution compared with $\chi^{2}=17.6$ with evolution.

The STAR weak boson asymmetries [20] are presented in the left-hand and center panels of Fig. 2 as functions of boson rapidity. The asymmetries are compared with model calculations [23] based on SIDIS and assuming no TMD evolution. The calculations are performed separately for different assumptions concerning the sign-change. The data show a preference for the model assuming the sign-change: $\chi^{2} / v=7.4 / 6$ with the sign-change compared to $\chi^{2} / v=19.6 / 6$ without. Hence, if the suppression from TMD evolution is small, the STAR data provide the first indication of the modified universality of the Sivers function.

\section{Conclusions}

Recent data collected by STAR have revealed the first transversity signals in polarized $p+p$ collisions and given tantalizing hints at the nature of QCD, such as insight into TMD factorization breaking and universality. Data collected in 2017 at $\sqrt{s}=510 \mathrm{GeV}$ promise more precise and diverse probes for expanding the frontier. The 2017 RHIC run represents an ambitious program targeting the Sivers sign-change through weak bosons, Drell-Yan, and direct photons, achieving $127 \%, 91 \%$, and $81 \%$, respectively, of the target integrated luminosities. The expected statistical precision of the weak boson and Drell-Yan asymmetries is shown in Fig. 3. The program probes the sign-change over a wide range of hard scales, which also enable the testing of TMD evolution. The 

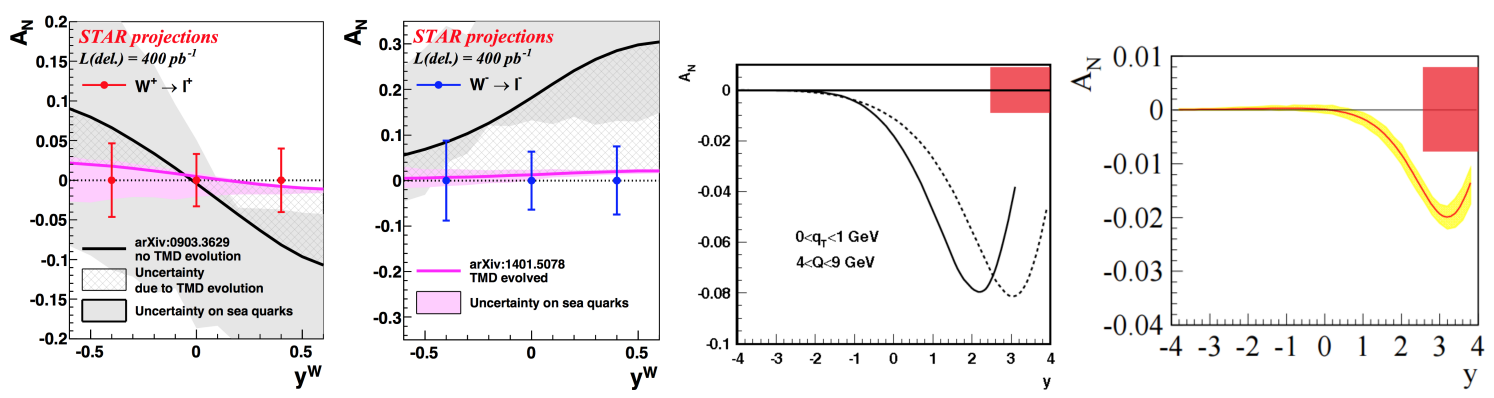

Figure 3: Projected statistical precision for weak boson and Drell-Yan transverse single-spin asymmetries from STAR data collected during the 2017 RHIC run at $\sqrt{s}=510 \mathrm{GeV}$. Projected weak boson statistics (far left and near left) are shown in comparison to model calculations from Refs. [23, 26], while those for Drell-Yan are shown in comparison to calculations from Refs. [26] (near right) and Refs. [27] (far right).

significantly enhanced precision of Collins asymmetries from 2017, in particular when combined with those from the 2015 dataset at $\sqrt{s}=200 \mathrm{GeV}$, will enable a more precise testing of TMD factorization breaking, universality, and evolution through the Collins fragmentation function. The 2017 run, therefore, stands to play a significant role in illuminating nucleon spin structure and the nature of QCD.

\section{References}

[1] E. R. Nocera, Impact of recent RHIC data on helicity-dependent parton distribution functions, in 22nd International Symposium on Spin Physics (SPIN 2016) Urbana, IL, USA, September 25-30, 2016, 2017, hep-ph/1702.05077.

[2] M. Anselmino, M. Boglione, U. D’Alesio, S. Melis, F. Murgia and A. Prokudin, Simultaneous extraction of transversity and Collins functions from new SIDIS and $e^{+} e^{-}$data, Phys. Rev. D 87 (2013) 094019, [hep-ph/1303.3822].

[3] Z.-B. Kang, A. Prokudin, P. Sun and F. Yuan, Extraction of quark transversity distribution and Collins fragmentation functions with QCD evolution, Phys. Rev. D 93 (2016) 014009, [hep-ph/1505.05589].

[4] J. Collins, Fragmentation of transversely polarized quarks probed in transverse momentum distributions, Nucl. Phys. B396 (1993) 161, [hep-ph/ 9208213$].$

[5] M. Radici, A. Courtoy, A. Bacchetta and M. Guagnelli, Improved extraction of valence transversity distributions from inclusive dihadron production, JHEP 05 (2015) 123, [hep-ph/1503. 034 95].

[6] J. C. Collins, S. F. Heppelmann and G. A. Ladinsky, Measuring transversity densities in singly polarized hadron hadron and lepton - hadron collisions, Nucl. Phys. B420 (1994) 565, [hep-ph/9305309].

[7] J. C. Collins, Leading twist single transverse-spin asymmetries: Drell-Yan and deep inelastic scattering, Phys. Lett. B 536 (2002) 43, [hep-ph/ 0204004 ].

[8] D. Sivers, Single spin production asymmetries from the hard scattering of point-like constituents, Phys. Rev. D 41 (1990) 83. 
[9] T. C. Rogers and P. J. Mulders, No generalized TMD-factorization in hadro-production of high transverse momentum hadrons, Phys. Rev. D 81 (2010) 094006, [hep-ph/1001.2977].

[10] Z.-B. Kang, X. Liu, F. Ringer and H. Xing, The transverse momentum distribution of hadrons within jets, hep-ph/1705.08443.

[11] A. Bacchetta and M. Radici, Dihadron interference fragmentation functions in proton-proton collisions, Phys. Rev. D 70 (2004) 094032, [hep-ph / 0409174 ].

[12] STAR collaboration, K. H. Ackermann et al., STAR detector overview, Nucl. Instrum. Meth. A499 (2003) 624.

[13] STAR collaboration, R. Fatemi, Constraining quark transversity through Collins asymmetry measurements at STAR, AIP Conf. Proc. 1441 (2012) 233, [hep-ex/1206.3861].

[14] STAR collaboration, L. Adamczyk et al., Observation of transverse spin-dependent azimuthal correlations of charged pion pairs in $p^{\uparrow}+p$ at $\sqrt{s}=200$ GeV, Phys. Rev. Lett. 115 (2015) 242501, [hep-ex/1504.00415].

[15] STAR collaboration, L. Adamczyk et al., Azimuthal transverse single-spin asymmetries of inclusive jets and charged pions within jets from polarized-proton collisions at $\sqrt{s}=500 \mathrm{GeV}$, hep-ex/1708.07080.

[16] STAR collaboration, J. K. Adkins and J. L. Drachenberg, Azimuthal single-spin asymmetries of charged pions in jets in $\sqrt{s}=200 \mathrm{GeV} p^{\uparrow}$ p collisions at STAR, Int. J. Mod. Phys. Conf. Ser. 40 (2016) 1660040.

[17] STAR collaboration, M. J. Skoby, High precision measurement of transversity using di-hadron correlations in $p^{\uparrow}+p$ collisions at $\sqrt{s_{N N}}=500 \mathrm{GeV}$, Int. J. Mod. Phys. Conf. Ser. 40 (2016) 1660038.

[18] STAR collaboration, K. Landry, Measuring transversity in $p^{\uparrow}+p$ collisions with di-hadron correlations at $\sqrt{s}=200 \mathrm{GeV}$ at the STAR experiment, in APS April Meeting, 2015.

[19] M. Cacciari, G. P. Salam and G. Soyez, The anti- $k_{t}$ jet clustering algorithm, JHEP 04 (2008) 063, [hep-ph/0802.1189].

[20] STAR collaboration, L. Adamczyk et al., Measurement of the transverse single-spin asymmetry in $p^{\uparrow}+p \rightarrow W^{ \pm} / Z^{0}$ at RHIC, Phys. Rev. Lett. 116 (2016) 132301, [nucl-ex/1511.06003].

[21] M. Radici, A. M. Ricci, A. Bacchetta and A. Mukherjee, Exploring universality of transversity in proton-proton collisions, Phys. Rev. D 94 (2016) 034012, [hep-ph/1604.06585].

[22] M. Radici and A. Bacchetta, Update on extraction of transversity PDF from inclusive di-hadron production, in Proceedings of the 25th International Workshop on Deep Inelastic Scattering and Related Topics (DIS 2017), 2017.

[23] Z.-B. Kang and J.-W. Qiu, Testing the time-reversal modified universality of the Sivers function, Phys. Rev. Lett. 103 (2009) 172001, [hep-ph/0903.3629].

[24] U. D'Alesio, F. Murgia and C. Pisano, Testing the universality of the Collins function in pion-jet production at RHIC, hep-ph/1707.00914.

[25] Z.-B. Kang, A. Prokudin, F. Ringer and F. Yuan, Collins azimuthal asymmetries of hadron production inside jets, hep-ph/1707.00913.

[26] M. G. Echevarria, A. Idilbi, Z.-B. Kang and I. Vitev, QCD evolution of the Sivers asymmetry, Phys. Rev. D 89 (2014) 074013, [hep-ph/1401.5078].

[27] Z.-B. Kang and J.-W. Qiu, Single transverse spin asymmetry of dilepton production near $Z^{0}$ pole, Phys. Rev. D 81 (2010) 054020, [hep-ph/ 0912.1319 ]. 